\title{
Labyrinthe
}

26 | 2007 (1)

Penser par extraordinaire

\section{«Pensez, dix minutes»}

Entretien réalisé par Marc Aymes et Déborah Cohen

Daniel S. Milo

\section{(2) OpenEdition}

Journals

Édition électronique

URL : http://journals.openedition.org/labyrinthe/1555

DOI : $10.4000 /$ labyrinthe.1555

ISSN : 1950-6031

Éditeur

Hermann

Édition imprimée

Date de publication : 15 mars 2007

Pagination : 79-101

ISBN : 978-2-9526131-3-2

Référence électronique

Daniel S. Milo, « «Pensez, dix minutes» », Labyrinthe [En ligne], 26 | 2007 (1), mis en ligne le 01 avril 2009, consulté le 20 avril 2019. URL : http://journals.openedition.org/labyrinthe/1555; DOI : 10.4000/ labyrinthe. 1555 


\title{
«PENSEZ, DIX MINUTES»
}

\author{
Entretien \\ avec Daniel S. Milo \\ réalisé par Marc AYMES et Déborah COHEN
}

\begin{abstract}
Connaissez-vous Daniel S. Milo? Il dit lui-même être «le seul témoin ici de [ses] propres exploits ». Il conviendrait donc de faire les présentations d'usage. Mais suffit-il de dire que l'homme est historien, romancier et philosophe, maître de conférences (habilité à diriger des recherches) à l'École des hautes études en sciences sociales à Paris ? Difficile, car malgré tout on en vient vite à s'écrier, sur le ton de parents excédés par l'insubordination de leur rejeton: «Qu'est-ce qu'il a encore fait?» Plutôt que de s'en remettre à cette normativité, laissons-le parler. Et contentons-nous d'un exergue, pour laisser voir l'une des raisons de l'entretien publié ci-après :
\end{abstract}

Écrire la Loi sur le dos des hors-la-loi, édifier la règle à base d'aberrant, définir la normalité avec du pathologique sont des procédés vieux comme le cerveau. L'extraordinaire, parce que meilleure Gestalt que l'ordinaire, attirera immanquablement notre attention, de lui on partira pour embrasser l'ensemble ${ }^{1}$.

LABYRINTHE - Commençons. Votre nom?

DANIEL S. Milo - C'est très policier tout ça! Daniel Shabetai Milo.

LABYRINTHE - Et que faites-vous dans la vie?

DANIEL S. MiLO - Jusqu'il y a deux ou trois ans, j'étais père et philosophe. Maintenant, je suis plutôt philosophe et père. Mes filles sont trop grandes même pour un père à mi-temps. Mais dans ma tête je suis toujours

1. Daniel S. Milo, «Épicerie, paranoïa, iceberg (épi)», dans Martine Godet (dir.) (avec la collab. de Muriel Carduner-Loosfelt et d'Hélène Coq-Lossky), De Russie et d'ailleurs. Feux croisés sur l'histoire. Pour Marc Ferro, Paris, Institut d'études slaves, 1995, p. 591-598, ici p. 592. 
père. Et fils orphelin. Ce n'est pas par hasard que je le dis. C'est une vision du monde verticale que j'ai. Toujours. Les liens forts que j'arrive à nouer avec l'univers sont des liens verticaux.

LABYRINTHE - Avez-vous eu une vie avant la recherche, si vous avez une vie de recherche? Car vous avez dit, lors d'un de vos séminaires, que "les étudiants d'aujourd'hui ayant une espérance de vie d'environ cent quatre-vingt dix ans, il vaudrait mieux qu'ils aient une vie avant...»

DANIEL S. MiLO - J'ai compris. Oui. J'ai eu une vie de romancier nul. J'ai fait trois romans avant d'aller à l'Université. Tous non publiés, heureusement. Tous une honte. Et puis... J'ai fait la guerre, j'ai été soldat. J'ai tiré sur des gens que je n'avais jamais vus avant. Ils m'ont tiré dessus aussi, alors qu'on n'avait pas particulièrement de comptes à régler. Estce que j'ai eu une autre vie... J'ai perdu un frère. C'était avant la recherche. Et d'ailleurs avant la recherche, il y a l'Université. En Israël j'ai fait des études de philosophie et de littérature, en deuxième cycle j'ai fait une maîtrise de cinéma. Puis, en troisième cycle - qui pour moi est avant la recherche, même si c'est un doctorat - j'ai fait ce que les autres appelaient «historien». Moi j'acceptais, avec ma tolérance habituelle. Ça c'était avant la recherche, la vraie. Avant ce que je fais maintenant, depuis 1990.

LABYRINTHE - C'est en 1990 seulement que vous avez commencé à faire de la recherche?

DANIEl S. Milo - Disons qu'à trente-sept ans je commençais à trouver. Un peu comme le rabbin Akiva. Ça vous dit quelque chose, Rabbi Akiva? Un berger analphabète jusqu'à quarante ans, il est devenu l'un des piliers du Talmud. Il est tombé amoureux de la fille de son patron qui a décidé que, s'il voulait l'épouser, il devait faire un peu d'études. Et il est devenu un grand rabbin. Mais si je compte ma vie vraie, c'est jusqu'à vingt-deux ans, et après trente-sept. Entre les deux se trouve la parenthèse universitaire normale, qui pour moi n'est pas une vraie vie. C'est une sorte d'inertie carriériste, arriviste, qui ne compte plus dans mon $\mathrm{CV}$ vrai.

LABYRINTHE - Aujourd'hui, vous n'êtes donc pas à l'Université, ni dans l'Université...

DANIEL S. Milo - Non, non. Je suis payé par le ministère, ce qui est très gentil. La France est un pays très accueillant pour les intellectuels 
étrangers, pour la main-d'œuvre immigrée intellectuelle. Le contribuable français règle mes notes. Ça s'arrête là. Je n'ai pas de contact avec le monde universitaire, ni ici ni en Israël, et cela depuis les colloques que j'ai organisés pour Alter Histoire ${ }^{1}$ - un à Viterbe, un à Royaumont, un à Paris - trois réunions où ça chahutait ferme.

Depuis 1989, je n'ai jamais mis les pieds dans un colloque. Je ne suis invité nulle part, je n'invite personne. Dans mon séminaire ${ }^{2}$, c'est moi qui parle. Le mois prochain j'invite quelqu'un, un cardiologue, mais c'est très rare; ça m'arrive à peu près tous les quatre ans. Donc, je vis dans... mon trou.

\section{LABYRINTHE - Et vous lisez quand même?}

DANIEL S. Milo - Il y a un dicton en hébreu : «Il faut choisir dans la vie, soit on fait la queue des lions, soit on fait la tête des renards.» J'ai compris qu'en pratiquant mes collègues, les historiens, les sociologues, les anthropologues, je faisais la «tête des renards». Alors qu'avec Platon et des grands romans, je faisais la «queue des lions». À l'âge de trentesept ans, j'ai fait mon choix. D'où le titre de mon séminaire : «Lire». Ça a commencé en 1989. C'était le challenge: être avec les grands esprits. Quand je dis «lire», c'est: on lit de grands textes. Des gens qui savent écrire, et non pas qui savent écrire en tant qu'historiens. De Marc Bloch, on dit: «C'est quelqu'un qui écrit bien pour un historien.» Mais quand on parle de Victor Hugo, on ne dit pas : «Il écrit bien pour un écrivain »! Il écrit bien, point. Donc chez moi, on lit des textes bien écrits, point. Shakespeare, Hawthorne, la Bible. Mais les textes d'historiens, les textes sociologiques...

LABYRINTHE - C'est aussi le choix de ne pas lire les contemporains?

DANIEL S. MiLo - Mais je lis des contemporains! James Ellroy, Saramago, des gens comme cela. Mais des historiens, ça c'est terminé. L'histoire, je ne peux plus la digérer du tout. Ce n'est même pas l'indigestion, ça ne m'intéresse plus.

1. Daniel S. Milo, Alain Boureau (dir.), Alter Histoire. Essais d'histoire expérimentale, Paris, Les Belles Lettres, coll. «Histoire», 1991. Sauf précision contraire, les ouvrages cités ci-après ont été publiés par le même éditeur.

2. À l'École des hautes études en sciences sociales (EHESS) à Paris, Daniel S. Milo y donne deux séminaires intitulés, pour l'année 2006-2007, «Le trop et le cerveau» et «L'expérimentation en sciences de l'homme». Dans la suite de l'entretien, il sera fait référence indistinctement à «l'École» ou aux «Hautes Études» pour désigner l'EHESS. 
LABYRINTHE - Et les philosophes?

DANIEL S. MILO - Platon.

LABYRINTHE - Et les philosophes contemporains?

DANIEL S. MILO - Platon. Il est très contemporain. Il est même beaucoup plus contemporain que la plupart des philosophes d'aujourd'hui. Je ne sais pas si vous avez vu une pièce de théâtre qui s'appelle La Dernière Mort de Socrate $e^{1}$ c'est une façon pour moi d'être, avec l'ami Socrate, mon unique maître.

LABYRINTHE - Et quel serait votre style dans tout cela?

DANIEL S. MiLO - Le style écrit, ou le style en général?

LABYRINTHE - En général, pour l'instant.

DANIEL S. Milo - Donc vous me posez la question «Quel est mon même?», n'est-ce pas ?

Quel est mon même? J'aime la vérité... follement. D'une façon maladive. C'est mon style. C'est un amour complètement absurde, qui ne sert à rien. L'amour de la vérité, c'est comme l'amour du camembert. Ce qui m'anime, c'est la faim de vérité.

Ce que j'aime dans la vérité, c'est que c'est toujours à sens unique; comme avec la femme fatale. Tout ce qui est unilatéral me plaît déjà. Tout ce qui est réciproque m'inquiète. La plupart des sciences humaines et sociales reposent sur le don/contre-don. Il y a énormément de variations sur le thème, mais c'est parce que l'humanité dans ses aspects sociaux, c'est give and take: donnant-donnant. Tout cela m'énerve terriblement depuis toujours. J'aime le don, tout seul, j'aime le give ou le take, mais pas les deux ensemble. La Vérité, c'est 2 x 2 font 4 . C'est l'idéal-type, pour parler comme Max Weber. Ce qui est propre à cette équation, 2 x 2 font 4 , ce qui est son même, c'est qu'il n'y a pas de dialogue entre elle et moi. Il n'y a pas de dialogue avec la vérité. En ce sens, je considère que je suis assez juif, assez jéhoviste. À savoir que la vérité ressemble au Un, ressemble à ce Dieu avec lequel on n'a pas de contact, et qui n'a pas besoin de nous. Il est là tout seul. Que j'apparaisse sur Terre ou pas, $2 \times 2$ font 4 n'est ni impressionné ni au courant. Tout ce qui est unilatéral en général, c'est mon truc; et à l'intérieur de cela, le lien maladif

1. Daniel S. Milo, (2002). 
qu'on a avec la vérité, qui est un cas spécifique de cet objet qui se passe si allégrement de nous. Pour l'amant de la vérité, il n'y a pas de récompense autre que l'amour qu'on a pour $2 \times 2$ font 4 . On ne peut pas toucher les dividendes de cet objet. On ne fait pas couple avec la vérité. C'est très asymétrique, l'amour de la vérité. La vérité n'aime pas l'amoureux de la vérité. Parce que si la vérité l'aimait - c'est comme pour Dieu -, elle ne serait pas vraie, car elle serait imparfaite, n'est-ce pas? C'est le modèle, c'est la théologie juive, si jamais une théologie juive existait.

Voilà: ça, c'est mon même. Est-ce que c'est mon style? J'en avais deux. J'en avais un avant la mort de ma mère, qui était violent, dans la provocation presque permanente. Quand j'allais dans les séminaires à l'École, il y avait des ambulances à la sortie pour venir chercher les orateurs. Au lycée aussi, il fallait que je descende celui qui parle. J'ai une haine viscérale de l'autorité, ou même de l'autorité provisoire. Quelqu'un parle, donc il faut lui tirer dessus, tout de suite. Donc, c'était un style un peu «fou furieux».

LABYRINTHE - Était-ce une manière de don? D'offrir son opposition?

DANIEL S. MiLO - Si l'on fait dans la psychanalyse, c'était une manière de tuer mon pauvre père. Mais on ne fait pas dans la psychanalyse. C'était pour moi un moyen de ne pas mourir d'ennui. Comme j'aime l'unilatéral, je suis assoiffé de grandeur. Or, les chances qu'on croise la grandeur à l'Université ou aux Hautes Études sont infimes, voire inexistantes. Donc j'en voulais à ceux qui parlaient d'être là, ex cathedra, d'être entre OK et médiocre. Ils n'arrivaient pas à me donner ce qu'il me fallait, ce que je trouve dans la littérature, dans Platon et dans Shakespeare, chez Johan Cruyff ou dans Don Giovanni. Donc c'était une sorte de rage: une façon d'être tout le temps dans le clash, dans le conflit. Ça, c'était mon style.

Autant, je n'accepte pas dans mon séminaire que quelqu'un ouvre le bec. Ça fait des années que c'est la règle écrite: chez moi on se tait. Les étudiants le savent. Ils ont le droit de poser des questions parce qu'ils n'ont pas compris. Mais ce n'est pas un dialogue entre nous. Jamais. Il ne faut pas que s'instaure un dialogue entre le maître et les disciples. Je leur dis: «Si je suis payé, c'est que je sais quelque chose que vous ne savez pas, donc je ne vois pas pourquoi on devrait échanger idées et opinions. » Donc, c'est sans dialogue. C'est même sans conflit - rien. Si quelqu'un me dit: «Je pense autrement», je dis : «Eh bien, allez chez 
mes collègues ». Parce que dans mon séminaire, on ne pense pas autrement. Très souvent, on ne pense rien. Mais pendant deux heures, vous êtes là, vous essayez de nager dans une pensée, ce n'est pas la peine de penser autrement. On essaie de comprendre la pensée qui se passe dans cet îlot, ce ghetto bizarre qu'est mon séminaire. Pour affiner ma réponse: s'il y a un même à l'intérieur de moi, c'est la violence. Toujours. Quel est mon style? Il est violent.

LABYRINTHE - Vous disiez que vous avez eu deux styles, non?

DANIEL S. Milo - La question, c'est: où est-ce que j'investis ma violence. Jusqu'en 1990, comme j'ai fréquenté plusieurs séminaires aux Hautes Études, comme j'ai fondé cette Alter Histoire, la violence était dirigée surtout contre les autres. C'était avant la mort de ma mère. J'étais toujours violent, c'est mon même. Depuis que j'ai souvenance de mon existence, je suis violent. Violent intellectuellement mais aussi violentviolent. On ne va pas en parler, mais violent: donner des coups, prendre des coups. La violence physique est aussi quelque chose qui m'attire, et que j'attire. Il ne faut pas le cacher. Ce n'est pas terminé: cinq policiers israéliens m'ont cassé les deux bras en 2002, dans les Territoires [palestiniens]. En 1990, quand le groupe Alter Histoire s'est évaporé, j'ai décidé d'investir ce même plutôt dans moi-même. Toujours violent, mais au lieu d'être sadique, être masochiste.

Après la mort de ma mère, je me suis beaucoup calmé. Certes, je reste plus violent que la moyenne nationale, et internationale, et intellectuelle ! Mais je souris plus, curieusement. Je suis tellement effondré par la mort de ma mère, tellement abattu... que je souris. Avec Laura, ma fille cadette, on se dit parfois: «Rien de grave ne peut plus nous arriver, puisque le pire est derrière nous.» Ça vous calme. Parce qu'avant, le pire était une possibilité. On pouvait le provoquer pour qu'il vienne. Maintenant le pire est derrière. Voilà, j'ai terminé ce discours.

\footnotetext{
LABYRINTHE - Et ne vous êtes-vous jamais dit que vous en faites un peu trop?

DANIEL S. Milo - Si, si, bien sûr. Beaucoup trop. Mais là-dessus, je suis un amateur de Thomas Bernhard... Vous l'avez lu? Il dit que tout ce qui compte dans l'histoire de l'humanité, même si c'est une histoire naturellement absurde (comme je le montre dans mon séminaire), est né de l'exagération. L'exagération paraît - aux yeux certainement de la
} 
sagesse parisienne française - pathétique? Alors je vote pour le pathos. Ça paraît ridicule? Alors je vote pour le ridicule. Avec cet espoir - dont vous parlez un peu dans les premières ébauches de votre texte «Pensez par extraordinaire ${ }^{1} \gg-$ avec cet espoir, certainement vain, mais qui n'est jamais mort, que du pathos, du ridicule et de mon intelligence, va naître le sublime, ou le miracle. Parce que je vois mal comment le miracle naîtrait autrement que de la cuisse du pathos. Je ne connais pas d'exemple autre. Donc, là je suis assez bernhardien, ou kafkaïen. Kafka aussi en a fait trop. Comme je l'ai écrit dans $\mathrm{Clefs}^{2}$, être Kafka c'est de la mégalomanie. Écrire La Colonie pénitentiaire, c'est déjà mégalomane. Et Dostoïevski, c'était l'exagération incarnée. Ce n'était pas possible d'imaginer un type comme Dostoïevski. Il faut lire les mémoires de Tolstoï, tellement jaloux de Dostoïevski parce qu'il était, lui, assez normal.

LABYRINTHE - Et en cinéma, avez-vous éprouvé ce genre de miracles $d u$ pathos?

DANIEL S. MiLO - Quand j'ai fait des films?

LABYRINTHE - Oui, ou quand vous en aimez.

DANIEL S. Milo - J'ai fait trois films. Comme pour le roman, je ne suis pas doué pour le cinéma. Je suis « correct +». Je les ai faits parce que je ne pouvais pas autrement. Il y en a un qui est exagérément pauvre, côté budget. L'écriture revient peu cher, alors que quand on fait un film qui coûte 1000 , ça se voit.

Cela dit, je vois très peu de films. Quand j'ai terminé mon mémoire sur le cinéma, en 1979, j'étais rassasié: j'avais vu deux ou trois films par jour pendant une année! Overdose. Alors que je ne me rassasierai jamais de la littérature. Je ne peux pas vivre sans romans, mais je peux vivre sans films. Et à l'intérieur de la littérature, alors là, le pathos, le ridicule, la prise de risque sont essentiels. Shakespeare!

LABYRINTHE - Dans l'«Avant-Manifeste» signé par Alain Boureau au début du livre Alter Histoire, il est question entre autres $d u$ « rejet de l'autorité des spécialistes et des techniciens», avec une parenthèse qui

\footnotetext{
1. À sa demande, nous avions transmis à Daniel S. Milo plusieurs extraits d'une version préparatoire du texte ci-inclus.

2. Paru en 1993, puis réédité dans Le Coffret (1997), qui comprend également Pour Narcisse, traité de l'amour impartial (1996) et Héros et cobayes (1997).
} 
explique qu'il s'agit du «refus des techniciens mais non des techniques que nous étions prêts à subtiliser ». Il est aussi question d' "un agacement devant les tropismes interdisciplinaires, vite chargés d'assumer seuls la réflexion historienne ${ }^{1}$ ». Ce qui nous a frappés, c'est que ces impulsions, ces mouvements, ont une résonance assez singulière avec des discussions ou des arguments que l'on a pu trouver sous la plume de tel ou tel dans Labyrinthe ces dernières années ${ }^{2}$ : d'un côté, un rejet d'une posture spécialiste, d'une autorité du spécialiste, et en même temps quelque chose qui diffracte, avec un trouble au niveau du lieu commun de l'interdisciplinarité. D'où l'adoption chez nous d'un mot comme «indiscipline», censé nous sortir de là. La question est donc la suivante: au total, tout cela ne vous donne-t-il pas un sentiment de déjà vu, de revenir au même, de réversion? Cela ne vous rend-il pas réticent et défiant, par certains côtés?

Daniel S. Milo - Vous plaisantez? Je ne suis ni réticent ni défiant, que non! Jaloux plutôt. Je suis envieux. Un vieil envieux.

LABYRINTHE - Et cependant vous incluez Alter Histoire dans une période de non-recherche...

DANIEL S. MiLO - Parce qu'il y a histoire dedans... Je vais parler en mon nom parce que c'est moi qui ai pris l'initiative, qui ai écrit le manifeste $^{3}$ : j'avais la chance d'avoir fait des études en Israël, à Tel Aviv, dans le département qui s'appelait «Science littéraire». C'est un oxymore, mais enfin, les membres de ce département étaient un peu prétentieux, beaucoup, énormément, passionnément prétentieux - ce qui est pathétique, ce que j'aime. Ce que j'ai appris, qui m'est resté et qui reste toujours dans ce que vous appelez «ma vie de chercheur», c'est qu'on est spécialiste d'une question, pas d'un objet. Tout est là, dans un noyau de noisette [d'après l'anglais in a nutshell, NdlR]. Dans les sciences sociales et dans les sciences humaines, on est spécialiste d'objets. En 1975 - je venais d'entrer à l'Université -, un professeur a dit dès la première leçon: «De même qu'il n'y a pas de spécialiste de la vache dans la faculté de biologie qui est à côté, chez nous il n’y a pas de spécialiste

1. Daniel S. Milo, Alain Boureau (dir.), Alter Histoire, op. cit., p. 7-8.

2. Voir ainsi Laurent Dubreuil, «Alter, inter : académisme et postcolonial studies », Labyrinthe $n^{\circ} 24$ : «Faut-il être post-colonial?», 2006/2, p. 60-61.

3. «Pour une histoire expérimentale», art. cit. 
ni de Dostoïevski, ni d'Agnon, prix Nobel israélien de littérature, ni de Hugo, ni du XIX siècle, ça n'existe pas. On ne définit pas un champ de recherche à travers l'objet. L'objet vient toujours en deuxième!» C'est l'ethos qui m'a conditionné en 1975-1978. Quelques-uns de mes professeurs de l'époque savaient le russe, ils pratiquaient, enseignaient, traduisaient les formalistes russes - Chklovski, Tinianov, Eichenbaum, Jakobson, etc. Cette bande d'avant-garde, très proche d'Eisenstein, de Maïakovski, de Pasternak, avait une pensée transversale de la culture et de la littérature. Chez eux, on n'était pas spécialiste de Tolstoï, on se posait de bonnes questions et on mobilisait Tolstoï pour les aborder.

Il y a des questions valables parce qu'il est urgent d'y répondre, et il y a des questions valables parce qu'il y a suffisamment d'objets qui permettent d'y répondre. Heidegger distingue entre le fragwürdig et le fraglich: respectivement ce qui mérite d'être posé comme question et ce qui peut être posé comme question. Ma thèse, Aspects de la survie culturelle $^{1}$, n'avait pas d'objet. C'était une question: quels mécanismes font que seuls quelques-uns des protagonistes et des œuvres restent, et que les autres trépassent? Il y a un chapitre sur les noms de rue, un autre chapitre sur les traductions littéraires, un troisième sur les manuels scolaires, et j'en oublie. Il y a un chapitre sur la Comédie-Française. Donc il n'y avait pas d'objet. La même question devient une sorte de projecteur qu'on promène.

La division du travail dans les sciences sociales et humaines a donc toujours été pour moi un non-sens, et cela dès que je suis revenu en France en 1982. Dans le département d'histoire en Israël, c'est la même chose: on est médiéviste, on est moderniste. On doit être spécialiste d'une question, pas d'une période. En biologie, on n'est pas spécialiste d'un gène, ou d'une protéine: on est spécialiste d'un processus biologique. Après on peut le vérifier chez les zebrafish, parce qu'étudier cette question sur les zebrafish est beaucoup plus efficace... Zebrafish, vous connaissez? C'est un poisson qui est transparent. Donc on peut voir très facilement ce qui se passe à l'intérieur de ses entrailles. Quand on étudie le développement de l'embryon durant les quarante-huit premières heures de son existence, on étudie le zebrafish. Mais les embryologistes sont-ils des spécialistes du zebrafish? Ils ne sont pas investis sentimen-

1. Paris, École des hautes études en sciences sociales, 1986. 
talement dans le zebrafish, mais par une problématique. C'est cela qui m'avait animé à l'époque, déjà.

C'est différent chez mes autres compagnons de route d'Alter Histoire, en premier lieu Alain Boureau. Boureau est un médiéviste, un philologue; il aime les détails. C'est un vrai spécialiste. C'est pour cela que l'attirer avec moi, c'était un triomphe. Le seul de la bande qui n'était pas un spécialiste «à l'ancienne», c'est Hervé Le Bras, qui est resté mon complice jusqu'à maintenant. Tous les autres étaient des experts. Ils avaient, à l'intérieur de la division du travail, un champ. Et qu'est-ce qu'un champ? Un domaine réservé qui permet d'avoir des arguments d'autorité. «Eh! Avant de vous prononcer sur l'objet spécifique, il faut d'abord faire trois-quatre ans.» Exemple: pour étudier Platon, il faut maîtriser le grec, n'est-ce pas ? Comme si les embryologistes parlaient la langue des zebrafish... Non! S'il y a une question qui m'intéresse chez Platon, il y a cinq traductions, je vérifie et, en plus, si je ne suis pas sûr, je peux aller voir un expert qui sait le grec. Ce que j'ai fait quand j'ai écrit mon texte sur l'An Mil, sur l'invention de l'An Mil' ${ }^{1}$ je suis allé voir Le Goff et Boureau pour des questions de latin. Je ne connais pas le latin.

En fondant le groupe Alter Histoire, nous avons refusé la notion de contexte tel qu'il est pratiqué dans les sciences humaines et sociales. À savoir que le contexte est donné. Chez nous, disciples des formalistes russes, le contexte est créé par la question. Il est confectionné par la question. Quand j'ai montré à Mona Ozouf mon texte sur le calendrier républicain ${ }^{2}$, elle a dit: «Oui, c'est superbe; mais...». Le «mais» renvoie au fait que je ne sois pas expert ès Révolution française. De même je me suis permis de travailler sur les manuels scolaires au XIX ${ }^{\mathrm{e}}$ siècle sans être historien de la pédagogie ${ }^{3}$; sur les noms de rue sans être urbaniste ${ }^{4}$; sur les peintres français entre 1650 et 1750 sans être historien de l'art; et j'en passe. Tout a été publié dans des revues spécialisées, mais avec cette méfiance: on publie parce qu'on trouve ça bon, parce que ça nous apporte quelque chose, mais l'auteur n'est pas des nôtres. C'est une sorte de dilettante, mais très intelligent... Vous voyez ce que je veux dire? Les

1. «L'An Mil. Un problème d'historiographie moderne», History and Theory, 273 (octobre 1988), p. 261-281.

2. Voir Trahir le temps (Histoire), 1991.

3. «Les classiques scolaires», dans Pierre Nora (dir.), Les Lieux de mémoire, Paris, Gallimard, coll. «Bibliothèque illustrée des histoires», t. II, vol. III, 1986, p. 517-562.

4. «Les noms de rues», dans Pierre Nora (dir.), op. cit., p. 283-315. 
spécialistes, les experts des Annales ${ }^{1}$ et des Lieux de mémoire ont accepté les objets, ce qui est d'ailleurs élégant de leur part, mais le bonhomme derrière le texte n'a jamais été accepté. Jamais. Le Goff et quelques autres disent que j'ai écrit le texte le plus important sur l'An Mil et la notion de «millénaire», mais quand il y a eu les festivités de l'an 2000, personne ne m'a invité parce que je ne suis point médiéviste. Parce que ce qui m'a intéressé dans l'An Mil, c'est une question: comment prend-on le système métrique, qui est de l'arbitraire absolu, et le plaque-t-on sur le temps ? Comment décide-t-on que c'est le système métrique qui tranche, qui charcute le temps, comme chez le boucher? Et après cela, comment ce système métrique arbitraire, qui vient des mathématiques, qui vient des deux mains, comment ce système qui n'a rien à voir avec le temps, divise-t-il quand même les livres et les corporations d'historiens? C'est cela qui m'a fasciné. Autrement dit, comment l'arbitraire crée du social, ou crée du réel. Le choix de l'An Mil n'était pas une excuse. Je trouvais très amusant de voir comment on avait inventé l'An Mil. Mais les médiévistes ne pouvaient pas m'accepter... parce qu'en plus je dis que l'An Mil a été inventé à la fin du XVI ${ }^{\mathrm{e}}$ siècle, en 1597, donc très loin de leur chasse gardée...

Ma démarche et Alter Histoire sont nés de ce refus absolu de la division contextuelle préfabriquée des «sciences» humaines et sociales. Quelques-uns de mes ex-pairs ont un sens de l'humour assez acide, mais quand ils écrivent ils se prennent très au sérieux. Ils n'ont pourtant jamais guéri qui que ce soit, ni de la grippe, ni certainement du sida, c'est-à-dire qu'ils n'apportent pas grand-chose à l'humanité... Ils se prennent tellement au sérieux, alors que ce qu'ils font n'est même pas de la protoscience. Dans mon manifeste de 1986, j'ai proposé à quelques uns de mes complices d'alors de chercher à gagner le substantif. Parce que l'adjectif on l'a : humaines, sociales, etc. Mais le substantif science est complètement immérité. D'où l'idée, grâce au réexamen de la notion de contexte, de décontextualiser, de dépayser ${ }^{2}$ de la division du travail dans ce monde qui est le vôtre, je pense, mais qui n'est plus le mien...

\footnotetext{
1. Voir par exemple, «Pour une histoire expérimentale, ou la gaie histoire», Annales ESC, 1990, 45/3, p. 717-734. Ou bien encore, «La bourse mondiale de la traduction : un baromètre culturel ? », Annales ESC, 1984, 39/1, p. 93-115.

2. Alter Histoire est constitué de deux «dossiers», intitulés «Métaphore» et «Dépaysement». Ce dernier, associé par Daniel S. Milo à l'idée d'une "spécialisation sous suspicion» ( «Pour une histoire expérimentale...», art. cit., p. 43), repose sur l'idée de présenter des sources «brutes» à des histo-
} 
Mais dites-moi, comment se fait-il que dans le texte que vous m'avez envoyé, «Penser par extraordinaire», le mot «cerveau» n'apparaisse pas une seule fois? Qu'est-ce que ça veut dire, penser? Il y a une limite à l'immatérialité du questionnement. On est tous très spirituels, très métaphysiques, évidemment. On n'est pas des paysans, on ne travaille pas la terre, mais je trouve qu'un dossier qui a pour premier terme «penser» et où l'on ne trouve pas le mot «cerveau», le mot «cognition», ou le mot «perception», je trouve ça... Qu'est-ce qui se passe ? mes chers, qu'estce qui se passe? Vous faites fausse route. Car il y a des liens qui sont très organiques, presque «organeux», synonymiques, entre pensée et cognition. La pensée, c'est une expression de la cognition. Et je ne parle pas de l'expertise cognitiviste ${ }^{1}$. Je parle du sens commun cognitiviste. C'est dans ce sens-là que j'entends penser. Ce qui me manque et qui m'a toujours manqué entre 1982 et 1989, quand je vivais dans ce milieu des Hautes Études, c'est l'absence totale de sens commun. Et ce qui m'étonne, c'est que cela se passe en France, là où un Descartes, dans le Discours de la méthode, et surtout un Montaigne ont dit: «Moi je pars toujours de mon expérience personnelle. C'est une sorte de système immunitaire. Quand on me dit quelque chose que je n'ai pas éprouvé moi-même et qui n'est pas valable chez moi, ça m'inquiète, c'est certainement un peu faux.» Donc penser-cerveau n'est pas un couple qui fait appel aux experts: c'est un couple, point! Parce que nous pensons par le cerveau. Il suffit de s'allonger ici et de penser. Vous allez voir: pensez, dix minutes. Ensuite, écrivez ce que vous avez pensé. Vous allez voir: le statut de l'ordinaire, et même le dosage entre ordinaire et extraordinaire dans dix minutes de pensée... Cela, c'est l'école de Montaigne. Montaigne n'est pas un expert, c'est quelqu'un qui se dit toujours contreexpert. Il détestait l'expertise. Il l'a dit: «Je suis l'expert d'un seul objet: Michel de Montaigne.» Dans ce sens là, je réfléchis, je pense, je me dis: tiens, l'extraordinaire occupe une place prépondérante dans la pensée.

riens non-spécialistes de leur «contexte». Le texte introductif au dossier en pose ainsi les enjeux : «L'historien est quotidiennement confronté aux sources; il en résulte une certaine automatisation dans ces rapports. La familiarité des sources qu'il étudie fonctionne comme un mécanisme de défense contre la réflexivité, contre la surprise aussi. Le jeu que nous nous proposons de jouer était appelé à désautomatiser cette familiarité, ou, pour parler comme Michel Foucault, à la problématiser» (op. cit., p. 143, souligné dans l'original).

1. La cognition a fait l'objet, précisément en tant que champ de vraie spécialité en formation, d'un précédent numéro de Labyrinthe, ${ }^{\circ} 20,2005 / 1$. 
L'extraordinaire, c'est ce qui tranche avec l'ordinaire. Je pense très très rarement à l'ordinaire. Je pense au pic. Pour parler un peu médecine, la distinction entre tonique et phasique vous dit quelque chose? Le tonique, c'est votre vie normale; le phasique, ce sont les pics. Par exemple, quand vous faites un effort extraordinaire, le débit cardiaque peut passer de cinq à trente litres. Là, forcément, on pense au pic.

C'est cette introspection qui me manque, ce sens commun qui est omniprésent chez Pascal, Descartes et Montaigne, mais qui a disparu de cette École, et de l'Université. Avec l'introspection, on se regarde penser, on se regarde agir, on se regarde réagir, et après on commence à écrire, et après on regarde les autres. Mais d'abord, on se regarde. Et quand on se regarde avec ce trio «penser-par-extraordinaire», on s'observe. Cette patience, ce silence de l'observation de soi me manquent: ils font défaut aux sciences sociales et naturelles, aux sciences sociales et humaines. Je ne les y retrouve pas. Alors que dans la littérature, on voit tout le temps cette observation de soi! On a l'impression que ce sont les écrivains les vrais experts, parce qu'ils parlent de quelque chose qu'ils connaissent, qu'ils maîtrisent. Même si c'est Philip K. Dick qui écrit Les andrö̈des rêvent-ils de moutons électriques? (Blade Runner): il sait, il connaît le fantasme, il connaît l'hallucination et le LSD. Alors que là, dans les sciences humaines et sociales, on a affaire à des gens qui ont des techniques. On a l'impression qu'ils ne connaissent - de façon personnelle, sensuelle - pratiquement rien, parce qu'ils ne se sont pas observés comme l'objet premier des sciences humaines. On est, je suis, d'abord objet des sciences humaines. Et après, objet des sciences sociales, parce que je suis quand même un tout petit peu un être social, sur les marges. Donc je m'observe d'abord. Prenez Bourdieu, par exemple: à la fin de sa vie, il a commencé à s'observer un tout petit peu, mais avant il était une sorte d'entomologiste. Il regardait les fourmis s'agiter, mais lui était hors du coup. C'est fou!

LABYRINTHE - Tous les domaines sont-ils accessibles, ou cette définition-là de la vérité implique-t-elle une restriction des champs? Par exemple, en m'observant, moi, puis-je comprendre ce que c'est que d'être un ouvrier?

DANIEL S. MiLO - Mais oui, vous pouvez. D'abord, vous êtes un être humain à $95 \%$. Donc vous avez beaucoup de moyens de comprendre l'ouvrier, parce que vous partagez avec l'ouvrier $95 \%$. 
Dans les années 1980, quand j'ai lu Lucien Febvre sur la question de l'incroyance ${ }^{1}$, je me suis dit: mais c'est n'importe quoi, c'est vraiment n'importe quoi... il est impossible que des gens du XVI siècle aient été à ce point différents de moi. Je ne peux pas l'accepter. Ce ne sont pas des Martiens, ni des chimpanzés. Ce sont des êtres humains. Et l'idée - il suffit de lire le Talmud pour comprendre -, l'idée que la croyance soit un bloc, et qu'à l'intérieur de la croyance il n'y ait pas aussi l'incroyance, l'idée qu'à l'intérieur de l'orthodoxie il n'y ait pas aussi l'hétérodoxie, et même l'athéisme, c'est une absurdité absolue. De là vient le livre de Paul Veyne: Les Grecs ont-il cru à leurs mythes ?² Lucien Febvre, lui, dit qu'au XVI ${ }^{\text {e }}$ siècle, il était impossible d'être athée. Mais qu'est-ce qu'il raconte!? Comme si Lucien Febvre n'était pas humain. Comme si lui il était un historien des années 1920-1930, alors que les gens de l'époque de Rabelais étaient des Français de 1540 ! Non, on est d'abord des humains, avec pratiquement le même cerveau. Et ce cerveau est tellement complexe que, comme le dit le Talmud, le plus grand juste est aussi le plus grand mécréant. C'est évident: plus on est grand, plus on a des doutes. Or les historiens, tels que je les ai pratiqués, n'ont jamais projeté leur propre expérience au-delà du contexte. Si le contexte, mon contexte, c'est 1920, alors je suis Lucien Febvre: il connaît très bien Strasbourg entre 1920 et 1930. Il sait ce que pensent les Strasbourgeois en 1932. Mais un Albigeois en 1912, pour lui, est déjà un Martien. C'est n'importe quoi. Donc vous pouvez comprendre un ouvrier sans problème.

Imaginons que vous soyez psychiatre. Vous refusez d'accepter un patient ouvrier parce que son monde vous échappe ? Est-ce que vous refusez d'accepter Rabelais? Si Rabelais a une crise... Par une sorte de machine à remonter le temps ou à descendre le temps, vous avez Monsieur Rabelais dans votre service de psychiatrie. Vous le refusez? «Désolée, je ne peux pas traiter des gens du XVI siècle.» Mais il dit: «Écoutez, j'ai la schizophrénie, c'était pareil au XVI siècle, j'ai de l'angoisse, c'était pareil au XVI ${ }^{\mathrm{e}}$ siècle»... Vous pensez que l'angoisse était très différente au XVI ? Ou que le désordre bipolaire était très différent? Certainement Rabelais était-il bipolaire. Mais si vous pouvez traiter

1. Rabelais et le problème de l'incroyance au XVI' siècle : la religion de Rabelais, Paris, Albin Michel, 1942.

2. Paris, Le Seuil, 1983. 
Rabelais de bipolaire, alors pourquoi ne pas comprendre un ouvrier? Ce n'est pas du chinois! Donc vous pouvez comprendre les ouvriers. Sans problème.

Dans votre dossier, j'ai vu l'«autre». Cet «autre» m'a fait penser à une phrase que j'ai écrite - hélas! je me cite trop. Une phrase de Clefs: «Quand on a l'altérité facile, on en connaît la recette.» Et comme je suis le seul témoin ici de mes propres exploits, je peux vous dire que depuis l'âge de deux ans, je suis considéré comme très différent des autres, classé très «autre». Mais c'est la paresse des autres qui nous voit autre. Je vous ressemble comme deux gouttes de sperme. Cette altérité dont on parle tout le temps, ce «connaître l'autre», ce culte de l'autre, c'est encore une mode qui ne repose sur rien de cognitif. On a fait des expériences: on a pris des Éthiopiens, des Juifs Falashas, qui sont arrivés d'Éthiopie. Avant de voir les gens de l'Agence juive, ceux qui les ont trimballés d'Éthiopie en Israël, ils ont vécu comme à l'âge préhistorique : pratiquement pas d'eau, pas d'écriture. Ces hommes ont été décrits comme des sauvages. Aujourd'hui, il y a déjà parmi eux des docteurs en mathématiques, des avocats. Ils ont un cerveau, c'est vrai. L'homme de Néanderthal avait pratiquement le même cerveau que nous. Alors l'ouvrier, a fortiori. Je suis et j'ai toujours été universaliste. Cette altérité n'est pas possible. Le seul autre que je connaisse, qui est vrai autre, c'est l'enfant; seuls les enfants en très bas âge nous sont pratiquement indéchiffrables. Ce sont vraiment des autres pour nous. Mais pas l'ouvrier. L'ouvrier, c'est le même. L'enfant, le bébé, c'est un autre. À l'âge de trois mois, six mois, il nous échappe complètement. La rencontre entre l'adulte et l'enfant, c'est de la véritable incompréhension essentielle.

LABYRINTHE - Ce qui est frappant dans la manière dont vous approchez les enjeux de l'extraordinaire, et dans sa violence, c'est une forme d'élitisme, y compris vis-à-vis de soi-même: une façon de chercher en soi-même les instants et les possibilités d'être plus qu'ordinaire. Cet élitisme renvoie-t-il aussi à un enjeu politique de refus du nivellement, donc de l'égalitarisme?

DAnIEl S. Milo - Je suis élitiste, c'est évident. Je suis élitiste comme l'était Socrate, mon seul maître, au sens où je sais que tout être humain qui a autour de 103 de coefficient d'intelligence et qui n'est pas complètement crétin me comprendra si je lui parle normalement, en tête-à-tête. Et ça vaudra la peine pour lui. Je pars de l'hypothèse que ce que je fais 
et ce que je dis est vrai, rare, et que ça appartient à tout le monde. Sauf que les autres n'y ont pas accès.

J'ai fondé à Sderot, une petite ville du sud d'Israël, la même d'où vient Peretz, le misérable ministre de la Défense, un groupe qui s'appelle Shakespeare pour le peuple. Je suis socialiste, et même plus que ça. Pas seulement du côté financier mais aussi du côté culturel : c'est injuste que moi et les miens nous ayons accès à Shakespeare et pas les ouvriers. Évidemment, il se peut que des socialistes prélèvent beaucoup plus d'impôts, mais alors une partie de ces impôts sert aussi à diffuser Shakespeare. Est-ce de l'élitisme? C'est du nivellement par le haut. Il faut créer un consensus, mais un consensus socratique: les grandes questions appartiennent à tout le monde. Ce n'est pas par hasard que tous mes livres sont sur Internet ${ }^{1}$. C'est pour cela que mon séminaire est «ouvert à tout avide de vérité ${ }^{2}$ ». C'est dans ce sens-là que je suis élitiste. La vérité est unfriendly, très peu amicale et même violente, masochiste, mais elle appartient à tout le monde. Ça m'étonne qu'il y ait si peu de demande... Enfin non, ça ne m'étonne pas tant que cela, parce que la vérité est très très peu amicale.

Je trouve votre formule bien trouvée: je suis élitiste à l'intérieur de ma propre histoire de vie. Et à l'intérieur de ma vie, à l'intérieur de mes cinquante trois ans, je revendique très peu de moments. Le reste, c'est la plèbe. Pratiquement toute ma vie, c'est la plèbe. Il y a quelques pics, à la fois pics positifs et de... pas d'ignominie, mais assez nuls - donc des pics négatifs aussi. Ce sont là les extraordinaires représentatifs dont je me solidarise. C'est ça, le moi que je revendique. Le reste est interchangeable. Dans ce roman assez médiocre que j'ai écrit (mais le deuxième volet sera meilleur), il y a un robot qui s'appelle Pourquoi Moi. Chaque fois que je suis invité par d'autres ou par moi-même à faire quelque chose, et que ma réaction première est: «Eh, pourquoi moi? pourquoi pas un autre?», le robot la fait à ma place et moi, je reste dans le congélateur. J'ai calculé: on peut vivre comme ça trois millions d'années. Les occasions où le meilleur de mon moi est mobilisé sont rarissimes. Pratiquement tout ce que je fais, par exemple ce que je vous raconte aujourd'hui, le moi d'il y a vingt jours aurait pu le faire à ma place. Donc je fais appel à Pourquoi Moi pour qu'il vous accorde l'interview. Alors

1. Voir www.danielmilo.com (visité le 25 février 2007).

2. Telle est la formule qui, dans le livret des enseignements et séminaire de l'EHESS (p. 177 de l'édition 2006), indique le «niveau requis » pour assister à celui de Daniel S. Milo. 
qu'il n'aurait pas pu faire le séminaire d'aujourd'hui' ${ }^{1}$. Impossible. C'était la première fois que je le faisais. Selon ce modèle, je méritais de vivre ces deux heures aujourd'hui. Le reste, les deux heures que nous passons ici ensemble, c'est agréable, mais ce n'est pas l'élite de mon être. Ce n'est pas la plèbe non plus ; c'est la bourgeoisie - certainement pas la noblesse. C'est bien dit: je suis auto-élitiste. C'est très très bien vu, bravo!

LABYRINTHE - Mais alors quid des implications en termes d'enseignement? Être "avide de vérité», ça s'apprend. Il faut quand même quelqu'un pour expliquer aux crétins ou aux demi-crétins...

DANIEL S. MiLO - Je n'ai jamais dit ça. Ni crétins ni demi-crétins, ce n'est pas ma formule. Dans le judaïsme on appelle ça un «tinoq chenichba», un bébé fait prisonnier. Ceux qui n'ont pas encore accès à cette parole - pas la mienne, mais aussi la mienne - en sont privés par méconnaissance. Ce ne sont pas des crétins, au contraire; tout au plus des ignorants, à cause de l'Éducation nationale et internationale, à cause de leurs parents, à cause de ceci ou cela, mais ils ne sont surtout pas crétins. Au contraire. Je suis socratique. Je suis socratique dans le sens où, pour Socrate, tout humain était candidat au dialogue socratique - enfin, disons plutôt: au faux dialogue socratique, puisqu'il n'y a pas de vrai dialogue dans mon séminaire. Tout humain. C'est pour cela que je m'étonne de faire mon séminaire en salle 10 [dans les bâtiments de l'EHESS au 105, boulevard Raspail à Paris] et pas au Zénith. Comment se fait-il que j'aie douze-dix-huit personnes et pas cent mille comme Johnny?

LABYRINTHE - Si vous le pouviez, vous souhaiteriez faire école? Comme Socrate, comme Aristote?

DANIEL S. Milo - Non, pas Aristote. Socrate. C'est moi, l'école, mais une école où je suis maître, disciple, trésorier, financier, tout.

LABYRINTHE - Mais vous parlez tout seul, quand même.

DANIEL S. MiLO - Je parle tout seul, mais il y a des gens qui m'écoutent un peu.

LABYRINTHE - Et dans quelles conditions peuvent-ils prendre la parole?

1. L'entretien a été réalisé un lundi, quelques heures après le séminaire en question. 
DANIEL S. MiLo - Le principe, le deal qu'on a passé et que j'ai à la fois écrit et signé - ils obéissent -, c'est que si on vient chez moi, ce n'est pas la peine de défendre autre chose; soit on garde ses croyances pour soi, soit on va chez le voisin. Dans mon séminaire se joue un paradigme cohérent. Je n'irais quand même pas critiquer Maradona parce qu'il ne prend pas la balle avec ses mains comme Michael Jordan. Le «miloïsme» est peut-être une école marginale et farfelue. Mais à l'intérieur de cette école je suis Maradona, Cruyff et Platini. Ce n'est donc pas la peine de me critiquer au nom de Derrida... Parce qu'il y a 10-15 ans, on m'expliquait souvent : «Mais Derrida dit ceci ; mais Lacan dit cela.» Je répondais : «Écoutez, Derrida est encore vivant, allez écouter Derrida. Avec Derrida on a un gentleman's agreement: je ne lis pas Derrida et Derrida ne me lit pas.»C'est comme cela qu'on revient dans le calme. Je préfère ne pas introduire Derrida chez moi. Quand on vient chez moi, on sait que c'est un univers clos.

LABYRINTHE - C'est curieux que vous disiez qu'il y a une cohérence, alors même que quand on vous lit on a vraiment affaire à...

DANIEL S. MiLO - ... à une effervescence.

LABYRINTHE - ... à une volonté de fragmenter.

DANIEL S. MiLO - C'est parce que je ne suis pas encore assez doué pour agencer le tout en système. J'espère que mon ultime livre sera un livre pédagogique, didactique, une vraie méthode. C'était tellement neuf pour moi en 1993 [date de la parution de Clefs]: je laissais faire les phrases, qu'elles sortent. Je n'ai pas essayé de les dresser, de les mettre en arguments. On y trouve des micro-arguments, mais forment-ils quelque chose de systématique? Pas encore. Il y a l'esprit, mais ça s'arrête là. Me reste à écrire le grand livre: il fera 120 pages, il dira ce qui mérite d'être dit, et il sacrifiera la poésie sur l'autel de la lisibilité.

LABYRINTHE - La forme poétique était-elle donc un pis-aller?

DANIEL S. MiLO - Non, c'était un chapitre. Je ne sais pas si ce style existe encore en moi, il faut que je me mette à écrire pour savoir.

LABYRINTHE - Cependant, si l'on se place du point de vue de ce qu'entendent vos élèves ou vos... «disciples», on peut supposer qu'ils ont intérêt à avoir un certain sens de la langue, y compris pour 
comprendre les expressions qui relèvent d'une poétique non noble, voire d'une poétique argotique.

DANIEL S. MiLO - Oui, je sais. Je fais non pas l'élève, mais le perroquet de Foucault qui dit: "Voici mon livre, c'est comme une boîte à outils, faites-en ce que vous pouvez en faire. Vous utilisez le marteau comme tournevis: bravo! - pour vous écraser le crâne: bravo aussi!» Ma poésie est postmoderne, hélas! J'ai beau être antirelativiste, ma façon de m'exprimer est très postmoderne - ou rabelaisienne... Ce mélange des genres: l'argot et le très haut, highbrow et lowbrow ensemble, tout le temps, le base-ball qui va avec Aristote. C'est comme ça que ma tête fonctionne. Michel Desgranges ${ }^{1}$ dit que je suis l'auteur de la maison le plus difficile à traduire. C'est curieux, ce melting-pot du français... Comme je dis dans Clefs, l'étranger, ça parle à côté ou ça se tait. Pas la peine que je me batte avec Proust: je vais perdre, je suis perdant d'emblée. C'est une langue que j'ai inventée; un peu.

LABYRINTHE - Est-ce cette manière d'être à côté, d'être entre deux ou plusieurs langues qui permettrait de toucher aux enjeux de l'extraordinaire par une désautomatisation qui vient aussi de votre situation?

DANIEL S. MiLO - Je ne sais pas. En Israël je suis pareil. Et pourtant l'hébreu est ma langue maternelle - comme si c'était une langue qui pouvait être maternelle! C'est une langue tellement difficile. Non, j'étais comme ça avant de partir de chez moi. Peut-être cela tient-il à ma biographie: $\mathrm{j}$ 'ai grandi dans un milieu très militaire, mon père était colonel dans l'armée israélienne, mon parrain était Moshe Dayan². Yitzhak Rabin habitait en face. Donc, être anarchiste puis de gauche dans ce milieu... Enfin, c'est comme ça. Diogène a un mot que j'épouse: «Je ne suis pas fou, mais je n'ai pas votre tête.» Non, je ne suis pas fou, mais je n'ai pas la tête de la plupart des autres personnes. Je vois un peu à côté des choses. Est-ce la langue? Non. D'ailleurs, j'ai appris le français avec L'Équipe!

LABYRINTHE - Donc vous diriez que vous êtes monstrueux?

DANIEL S. MilO - Oui... oui... heureusement...

LABYRINTHE - Merci.

1. Directeur des éditions Les Belles Lettres jusqu'à fin 2006.

2. Haut responsable militaire et politique israélien, qui s'est illustré durant les guerres des Six Jours (1967) et du Yom Kippour (1973). 